\title{
PROPUESTA PARA LA MEJORA DE LAS FACILIDADES DE PRODUCCIÓN DEL CAMPO A
}

Carlos Alberto Cerquera Rojas ${ }^{1^{*}}$, Rubén Darío Conde León², Sergio Iván Villamizar Delgado ${ }^{3}$

* A quién debe dirigirse la correspondencia.

\section{RESUMEN}

El trabajo de investigación se focalizó en el diseño de una propuesta para la mejora de las facilidades de producción del Campo A, la cual da solución a la problemática de la alta recurrencia de fallas en las tuberías de producción por problemas de abrasión y corrosión, evaluando nuevas tecnologías ofrecidas en el mercado para dichas tuberías, a fin de reducir costos operacionales y mitigar riesgos de seguridad industrial. Para lograr el objetivo, se realizó una evaluación técnica y económica de la inversión de instalar tubería de producción revestida, y se obtuvo resultados favorables en cuanto a la reducción de costos y aumento de ventajas operacionales, para un campo con las condiciones y características del Campo A. Como conclusión, se obtuvo que el proyecto es financieramente viable. Lo anterior también indica que la inversión se recupera rápidamente y por tanto se recomienda realizar la inversión.

Palabras Clave: Corrosión; Tubería de Producción Tradicional; Tubería de Producción Revestida; Facilidades de Producción; Costos Operacionales.

\section{PROPOSAL FOR THE IMPROVEMENT OF THE PRODUCTION FACILITIES FIELD A}

\begin{abstract}
This research was focused on the design of a proposal for the improvement of the production facilities Field A, which solves the problem of high recurrence of failures in production pipelines because of the abrasion and corrosion problems, evaluating new technologies offered in the market for such pipelines in order to reduce operational costs and mitigate operational risks. In order to reach the aim, technical and economic evaluation of the investment to install production coated tubing was performed, obtained favorable results in terms of cost improvements and increase on the operational advantages for a field with the field conditions and characteristics like the Field A. As a result, the research concluded that the project is financially feasible. These also indicates that the investment is recovered quickly and therefore is recommended that the operator make the investment.
\end{abstract}

Keywords: Corrosion; Production Pipelines; Production Coated Tubing; Production Facilities; Operational Costs.

1. Vicerrectoría Académica. Universidad Surcolombiana, USCO, Avenida Pastrana Borrero con Carrera 1a., Neiva, Colombia. E-mail: calcero@gmail.com.

2. Ingeniero de Operaciones. Schlumberger Land Rigs (Saxon Services), Bogotá, Colombia. E-mail: rubencho1412@hotmail.com.

3. Departamento de Ingeniería Electrónica. Universidad Nacional de Colombia, UNAL, Carrera 45 No. 26 - 85 , Bogotá, Colombia. E-mail: sivillamizard@unal.edu.co. 


\section{INTRODUCCIÓN}

El objetivo del presente trabajo es investigar en detalle la forma de mitigar los problemas de corrosión y abrasión que se vienen presentando en las tuberías de producción tradicionales en pozos con fallas recurrentes, de campos con condiciones similares a las del yacimiento del Campo A. Lo anterior, mediante la evaluación técnica y económica de la instalación de tubería de producción revestida para reducir los costos operativos en los pozos con problemas de corrosión.

Se presume que con la instalación de la tubería revestida en las sartas de completamiento de pozos con problemas de corrosión, se alargará el tiempo de vida de estos materiales, disminuyendo la frecuencia de intervención y la inyección de químicos anticorrosivos. Adicionalmente, se espera mitigar riesgos ambientales y de seguridad industrial, de acuerdo a las alternativas actuales del mercado.

Para abordar la problemática se desarrollaron los siguientes procesos:

a. Evaluación de la situación actual de las facilidades de producción (tuberías de producción) de los pozos productores del Campo A.

b. Proponer una solución técnica para la disminución de la recurrencia de fallas en pozos del Campo A, evaluando nuevas alternativas de tuberías de producción, con el fin de reducir costos operacionales y mitigar riesgos ambientales y de seguridad industrial.

c. Realizar una evaluación económica - financiera de la propuesta anterior a fin de realizar el cambio de la tecnología de la tubería de producción.

\section{PROBLEMA}

Una de las principales fallas que se presentan en la infraestructura petrolera, y precisamente en las facilidades de producción, son las averías en las estructuras del completamiento entre las cuales se encuentran la tubería de producción en fondo, los cabezales de los pozos, las tuberías de superficie, y el equipo de procesamiento ubicado aguas abajo. Lo anterior, se debe primordialmente al proceso de corrosión, el cual comienza en el momento de fundición del acero y se acelera en los campos petroleros por causa de la presencia de especies ácidas - tales como el ácido sulfhídrico $\left(\mathrm{H}_{2} \mathrm{~S}\right)$ o el dióxido de carbono $\left(\mathrm{CO}_{2}\right)$ (Jaspe, 2006) - en muchos fluidos de formación, $\mathrm{y}$, adicionalmente, debido a las presiones $\mathrm{y}$ temperaturas elevadas de las formaciones productivas (Popoola, Grema \& Latinwo, 2013).

En un pozo petrolero ya completado, las consecuencias de la corrosión se enmarcan en la pérdida de resistencia, ductilidad, tenacidad y la reducción del espesor de las paredes del acero, componentes de los tubulares de fondo de pozo y demás herramientas que componen el completamiento. Lo anterior, se traduce en riesgos ambientales, de seguridad industrial y, complementariamente, en el aumento de los costos operacionales (OPEX), generado por las múltiples intervenciones que se deben realizar al pozo (Roberge, 2000).

El Campo A se completa mediante facilidades de fondo tales como ESP (electrosumergible pumps - por sus siglas en inglés) (Amorocho, 2006), a profundidades entre $7.000-9.000 \mathrm{ft}$ (dependiendo de la presión del pozo) (Castillo, 2005), y en algunos casos se completan con empaques hinchables. Igualmente, se usa tubería de producción convencional (tubing) de $31 / 2$ " y 2 7/8", conexión EUE (Colque, 2012).

En las tuberías de producción se identificó corrosión en las mismas, gracias a inspecciones rutinarias y Workovers realizados en los campos, causados principalmente por la abrasión y la presencia de $\mathrm{CO}_{2}$ (Nalli, 2010). Dicho problema se evidencia especialmente en las juntas de la tubería y cerca de la superficie. Para atacar el problema de corrosión, en muchos casos se han puesto en marcha la instalación de líneas de inyección química hasta en dos puntos: la bomba en el fondo del pozo y en superficie. Sin embargo, a pesar de la inyección de químicos en algunos pozos se sigue presentando dicho problema, afectando la tubería de producción.

Como consecuencia de este problema, la rentabilidad del proceso productivo y las metas financieras establecidas inicialmente en la empresa se ven afectadas negativamente de manera significativa, debido a la mayor cantidad requerida de intervenciones de inspección a las tuberías de producción. Hasta el momento, no se tienen grandes diferidas por causa del problema de corrosión en campo: sin embargo, los costos operativos se incrementan a raíz de las continuas reparaciones y cambios de bomba, ocasionados por problemas de corrosión.

Actualmente el problema de la corrosión en la industria de la producción de petróleo y gas está costando un monto anual estimado de USD \$1.372 millones, desglosados en USD $\$ 589$ millones en costos de tuberías de superficie e instalaciones, USD \$463 
millones anuales en gastos de tuberías de producción de fondo de pozo y otros (los cabezales de los pozos y el equipo de procesamiento ubicado aguas abajo) y USD \$320 millones en gastos de capital relacionados con la corrosión (Champion Technologies, 2012). Lo anterior, se debe primordialmente a la presencia de especies ácidas - tales como el ácido sulfhídrico $\left(\mathrm{H}_{2} \mathrm{~S}\right)$ o el dióxido de carbono $\left(\mathrm{CO}_{2}\right)$ - en muchos fluidos de formación, y a causa de las presiones y temperaturas elevadas de las formaciones productivas.

A la fecha, en el Campo A no se han presentado accidentes operacionales generados por la alta corrosión encontrada en las tuberías de producción. A pesar de ello, en algunos de los pozos ha sido necesario cambiar la línea de producción en superficie, debido a la posibilidad de presentar fugas y derrames. Dicho riesgo ha sido controlado preventivamente.

En el mercado, actualmente se están presentando algunas alternativas novedosas en las tuberías de producción, las cuales presentan revestimientos de polímeros, diversas aleaciones y materiales con mayor resistencia a la corrosión.

Con la instalación de tubería revestida en las sartas de completamiento de pozos con problemas de corrosión, se espera alargar el tiempo de vida del completamiento, buscando así disminuir la recurrencia de fallas en pozos debido a daños y la inyección de químicos anticorrosivos, en comparación con la tubería tradicional de producción.

Se tiene como objetivo reducir costos operacionales y mitigar riesgos ambientales y de seguridad industrial de acuerdo a las alternativas actuales del mercado.

\section{CORROSIÓN EN LA INDUSTRIA PETROLERA}

La corrosión en las tuberías utilizadas en los pozos de la industria del petróleo y gas se debe principalmente a la presencia de Dióxido de Carbono $\left(\mathrm{CO}_{2}\right)$, Sulfuro de Hidrógeno $\left(\mathrm{H}_{2} \mathrm{~S}\right)$ y la producción de agua en el fluido de producción (Lusk, Gupta, Boinapally \& Cao, 2008).

El $\mathrm{CO}_{2}$ usualmente está presente en los fluidos producidos. Aunque este, no puede por sí sólo causar el modo catastrófico de falla por grietas asociado al $\mathrm{H}_{2} \mathrm{~S}$. Sin embargo, su presencia en contacto con una fase acuosa puede resultar en altas velocidades de corrosión donde la morfología de ataque suele ser altamente localizada (corrosión mesa) (Kermani \& Morshed, 2003).
La corrosión del acero al carbono en ambientes con $\mathrm{CO}_{2}$ es un fenómeno muy complejo y aún requiere futuras dilucidaciones. Han sido propuestos muchos mecanismos para este proceso. Sin embargo, estos aplican para condiciones muy específicas o no han tenido amplio reconocimiento o aceptación.

En general, el $\mathrm{CO}_{2}$ se disuelve en agua para formar ácido carbónico $\left(\mathrm{H}_{2} \mathrm{CO}_{3}\right)$, un ácido débil comparado con los ácidos minerales ya que este no puede disociarse completamente.

Es evidente que las concentraciones de especies de $\mathrm{CO}_{2}$ disuelto en solución y el transporte de masa del $\mathrm{CO}_{2}$ disuelto a la superficie metálica tiene una influencia crítica en la reacción y subsiguiente velocidad de corrosión y que cada especie disuelta presente en el medio puede contribuir a la reacción catódica (Harrop, Martin \& White, 1988). Consecuentemente, hay una clara necesidad de caracterizar la química de la solución con respecto a la disolución del $\mathrm{CO}_{2}$ donde la acidificación resultante dependerá también de la composición del agua y de su capacidad buffer (Crolet \& Bonis, 1983).

La corrosión por $\mathrm{CO}_{2}$ ocurre principalmente en forma de corrosión general y en tres tipos de corrosión localizada: picaduras (Ver Figura 1), ataque mesa (Ver Figura 2) y corrosión localizada inducida por el flujo. En el estudio de la corrosión por $\mathrm{CO}_{2}$, se debe hacer una clara distinción entre la corrosión pura por $\mathrm{CO}_{2}$ y la corrosión por interacción combinada de la erosión y $\mathrm{CO}_{2}$, la cual puede aumentar la velocidad de corrosión por $\mathrm{CO}_{2}$. Esta última se evidencia en forma de marcas de ondulación, herraduras, colas de cometa, huellas de dinosaurio, etc. (Kermani \& Smith, 1997).

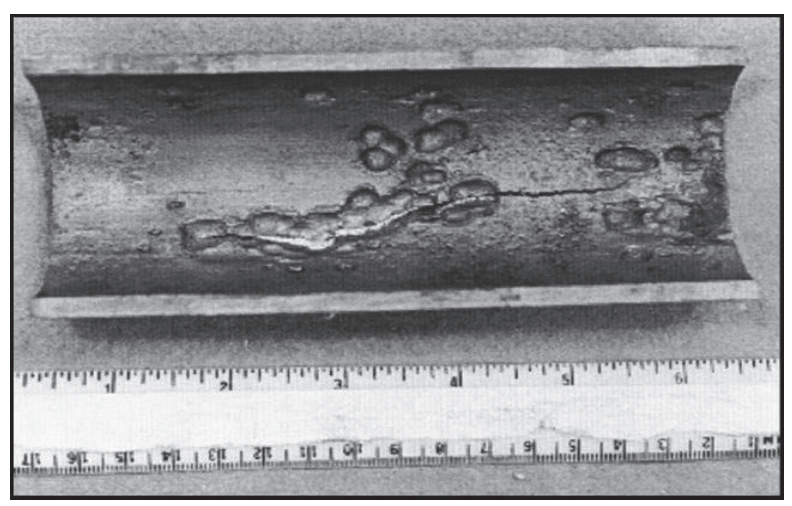

Figura 1. Picaduras

Fuente: Jaspe, R., U., R. (2006). Estudio de la resistencia a la corrosión por $\mathrm{CO}$, de aceros de tuberías $3 \% \mathrm{Cr}$ para pozos petroleros (fotografia). Recuperado de http://159.90.80.55/ tesis/000142075.pdf. 


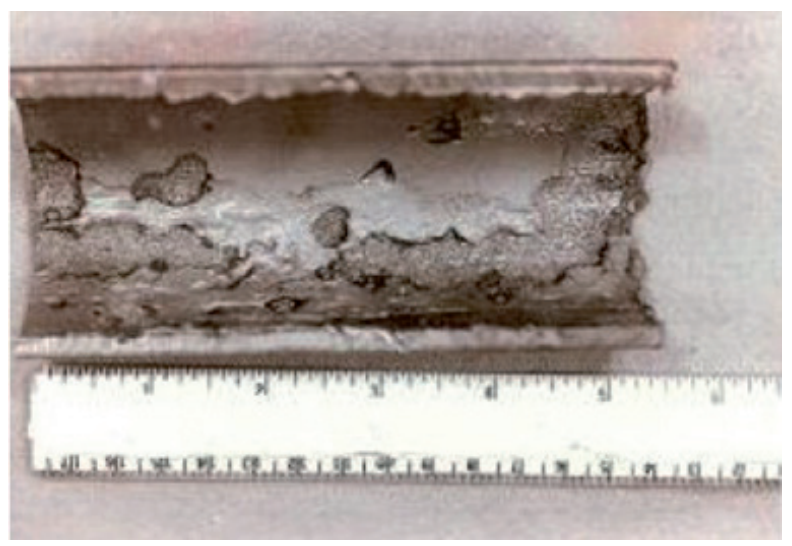

Figura 2. Ataque Tipo Mesa

Fuente: Jaspe, R., U., R. (2006). Estudio de la resistencia a la corrosión por $\mathrm{CO}_{2}$ de aceros de tuberías 3\% Cr para pozos petroleros (fotografía). Recuperado de http://159.90.80.55/ tesis/000142075.pdf.

El fenómeno de corrosión por $\mathrm{H}_{2} \mathrm{~S}$ en tuberías de producción de crudo, tiene lugar en presencia de sulfuro de hidrógeno $\left(\mathrm{H}_{2} \mathrm{~S}\right)$ y agua (Documento Técnico de PDVSA, 2008). Aunque de una forma más general se puede resumir la corrosión por sulfuro de hidrógeno como:

$\mathrm{H}_{2} \mathrm{~S}+\mathrm{Fe}+\mathrm{H}_{2} \mathrm{O} \rightarrow \mathrm{FeS}+\mathrm{H}_{2} \mathrm{O}+\mathrm{H}_{2}$. Como producto de la reacción se obtiene Sulfuro de Hierro (FeS) con desprendimiento de hidrógeno (Documento Técnico de PDVSA, 2008).

La corrosión por $\mathrm{H}_{2} \mathrm{~S}$ generalmente se presenta bajo las siguientes formas: a. Película de FeS y b. Picaduras distribuidas al azar de forma cónica y bordes filosos, de color negro o azul profundo (Ver Figura 3).

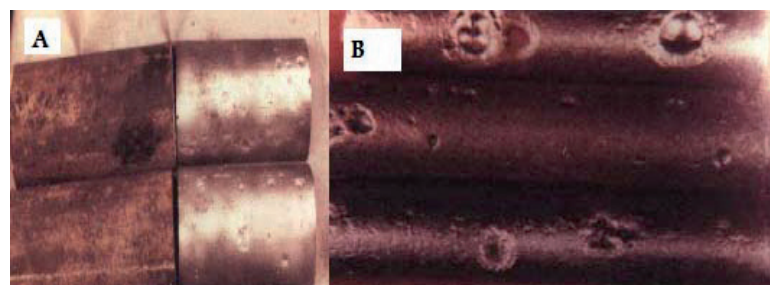

Figura 3. Corrosión por $\mathrm{H}_{2} \mathrm{~S}$. (A) Generalizada y (B) por Picaduras

Fuente: Guevara, M., M., F. (2010). Estimación del potencial incrustante y corrosivo en pozos productores y lineas de transferencia del Campo Dación, San Tomé (fotografía). Recuperado de http://ri.biblioteca.udo.edu.ve/ bitstream/123456789/2802/1/076-TESIS.IQ.pdf.
El gas de producción de los pozos es una mezcla de gases orgánicos con concentraciones considerables de $\mathrm{H}_{2} \mathrm{~S}, \mathrm{CO}_{2} \mathrm{y} \mathrm{Cl}^{-}$. La presencia de alguno de estos agentes, en un medio acuoso, es capaz de producir graves daños por corrosión, pero la combinación de los mismos a determinadas condiciones operacionales puede acelerar o disminuir la velocidad de corrosión (Documento Técnico de PDVSA, 2008).

La extracción continua de $\mathrm{CO}_{2}, \mathrm{H}_{2} \mathrm{~S}$ y agua a través de las facilidades de producción de petróleo y gas, con el tiempo hacen que las superficies internas de estos componentes comiencen a sufrir los efectos de la corrosión. Las líneas y los accesorios que componen las tuberías sufrirían degradaciones en los materiales debido a las condiciones variables del pozo tales como cambios en las composiciones de fluidos, acidificación de pozos durante el período de extracción y cambios en las condiciones de presión y temperatura de funcionamiento. Dicha degradación conlleva a la reducción de propiedades mecánicas tales como resistencia, ductilidad, resistencia al impacto, y así sucesivamente. Lo anterior conducirá a un punto en el cual el componente se puede romper completamente y se tendrá la necesidad de reemplazarlo, deteniendo la producción por completo. Las graves consecuencias del proceso de corrosión se han convertido en un problema de importancia en todo el mundo (Roberge, 2000).

Las causas de la corrosión interna en sistemas petroleros se deben al uso de aceros al carbono y de baja aleación en la construcción de tuberías, que además de ser materiales costo - efectivos, son susceptibles a corrosión. Muchos factores influencian la velocidad de corrosión, por ejemplo: la velocidad del flujo, las composiciones de aceros, el crudo y agua de formación, la temperatura, el pH y la humectabilidad del acero expuesto al medio (Papavinasam, Doiron, Panneerselvam \& Revie, 2007).

Si la superficie del metal se corroe generalizadamente, pero las capas de producto de corrosión no se depositan formando películas pasivas que cubren la superficie, entonces la corrosión general puede continuar.

$\mathrm{Si}$, como ocurre comúnmente, los productos de corrosión se depositan y forman capas que cubren la superficie, en este caso el acero es susceptible a corrosión por picaduras. El grado de susceptibilidad depende de la estabilidad de las capas, la temperatura, composición de la salmuera y flujo. La penetración a la pared de la tubería es un proceso que ocurre en tres etapas: a. 
Formación, crecimiento, ruptura y formación de capas sobre la superficie metálica; b. Iniciación de picaduras en regiones localizadas de la superficie del metal donde hubo ruptura de la capa de producto de corrosión y c. Propagación de la picadura y eventual penetración de la pared de la tubería (fuga) (Papavinasam, et al., 2007).

Estudios recientes han demostrado claramente que a pesar de la investigación extensiva en las pasadas cuatro décadas, la comprensión del mecanismo de corrosión por $\mathrm{CO}_{2}$ permanece incompleto. La existencia de modelos cuantitativos no es confiable en la predicción de la velocidad actual de aceros al carbono y de baja aleación, el cual invariablemente resultan en una sobre especificación de materiales e impacta adversamente los costos de producción de crudo y gas (Kermani \& Morshed, 2003).

De todas las fallas que ocurren en las operaciones de la industria del gas y del petróleo, la más importante es la Corrosión con el 33\% de los casos, seguido por la Fatiga con el 18\% y el Daño Mecánico con el 14\% de los casos, como puede verse en la Tabla 1.
Tabla 1. Tipos de Fallas que se presentan en la Industria Petrolera

\begin{tabular}{|lc|}
\hline \multicolumn{1}{|c}{ Tipos de Fallas } & \% \\
\hline Corrosión & 33 \\
\hline Fatiga & 18 \\
\hline Daño Mecánico & 14 \\
\hline Fractura Frágil & 9 \\
\hline Defecto de Fabricación & 9 \\
\hline Defectos de Soldadura & 7 \\
\hline Otros & $\mathbf{1 0}$ \\
\hline
\end{tabular}

Fuente: Jaspe, R., U., R. (2006). Estudio de la resistencia a la corrosión por $\mathrm{CO}_{2}$ de aceros de tuberías $3 \%$ Cr para pozos petroleros. Tomada de http://159.90.80.55/tesis/000142075. pdf. p. 1.

A su vez, el tipo de corrosión que ocurre se distribuye aproximadamente de la siguiente forma, por $\mathrm{CO}_{2}$ con el $28 \%$, por $\mathrm{H}_{2} \mathrm{~S}$ con el $18 \%$ y en Soldaduras con el $18 \%$, como puede verse en la Tabla 2.

Tabla 2. Tipos de Corrosión que se presentan en la Industria Petrolera

\begin{tabular}{|l|l|}
\multicolumn{1}{|c}{ Tipos de Corrosión } & \% \\
\hline Por $\mathrm{CO}_{2}$ & 28 \\
\hline Por $\mathrm{H}_{2} \mathrm{~S}$ & 18 \\
\hline En Soldaduras & 18 \\
\hline Picaduras & 12 \\
\hline Corrosión - Erosión & 9 \\
\hline Galvánica & 6 \\
\hline Espacios Confinados & 3 \\
\hline Impacto & 3 \\
\hline Corrosión bajo Tensión & 3 \\
\hline
\end{tabular}

Fuente: Jaspe, R., U., R. (2006). Estudio de la resistencia a la corrosión por $\mathrm{CO}_{2}$ de aceros de tuberías $3 \%$ Cr para pozos petroleros. Tomada de http://159.90.80.55/tesis/000142075.pdf. p. 2.

\section{TUBERÍA DE PRODUCCIÓN (TUBING)}

La tubería de producción es utilizada para la conducción de los fluidos bombeados desde el fondo del pozo hasta la superficie. Los criterios de selección de la tubería de producción están basados en los fluidos producidos y su velocidad de flujo, la profundidad donde se ubica la bomba y las características corrosivas de los fluidos.

El proceso de manufactura que la industria ofrece para este tipo de tubería es la soldadura por resistencia eléctrica (con costura) o tubería sin costura. El tratamiento térmico que se le practica a esta tubería generalmente es un normalizado (Cuadrado \& Prada, 2005).

Uno de los grados más empleados es el API J55 el cual posee una composición química similar al acero AISI SAE 1045. La norma API 5CT solamente específica para este acero porcentajes de fósforo y azufre máximo de $0,030 \%$ para cada uno. 


\section{CARACTERÍSTICAS ACTUALES DEL CAMPO A}

Las características de los pozos de referencia a los cuales se les van a cambiar la Tubería de Producción Tradicional por la Tubería de Producción Revestida se muestran en la Tabla 3.

Tabla 3. Características de los pozos de referencia a los que se les van a cambiar la Tubería de Producción

\begin{tabular}{|c|c|c|c|}
\hline & Temperatura & $\begin{array}{l}\text { Presión de } \\
\text { Cabeza de } \\
\text { Pozo }\end{array}$ & Caudal \\
\hline $\begin{array}{c}\text { Pozos de } \\
\text { Referencia }\end{array}$ & $207^{\circ} \mathrm{F}\left(97^{\circ} \mathrm{C}\right)$ & $\begin{array}{l}\text { Entre } 200 \mathrm{y} \\
300 \mathrm{lb} / \mathrm{pulg}^{2}\end{array}$ & $\begin{array}{c}\text { Entre } 5.000 \text { y } \\
9.000 \text { barriles } \\
\text { diarios de fluido, } \\
\text { de los cuales entre } \\
300 \text { y } 600 \text { BOPD }\end{array}$ \\
\hline
\end{tabular}

Número de pozos afectados con respecto al total de pozos del Campo: Actualmente en el Campo A existen 350 pozos productores, de los cuales el $20 \%$ (70 pozos) presentan problemas de corrosión por diferentes factores, producción de arena por diferentes factores, producción de arena y presencia de Dióxido de Carbono - $\mathrm{CO}_{2}$.

Fuente y el Tipo de Corrosión que se presenta: La corrosión en los campos petroleros se manifiesta por sí misma en varias formas, entre las más relevantes o comunes formas de ataque encontradas están: corrosión por Dióxido de Carbono $\left(\mathrm{CO}_{2}\right)$ o corrosión dulce, corrosión por Sulfuro de Hidrógeno $\left(\mathrm{H}_{2} \mathrm{~S}\right)$ o corrosión ácida, las dos anteriores presentes en los fluidos producidos y corrosión por Oxígeno en los sistemas de inyección de agua.

En un gran porcentaje, las fallas ocurridas en campos petroleros resultan de la corrosión por $\mathrm{CO}_{2}$ de aceros al carbono y de baja aleación primordialmente. Lo anterior debido al inadecuado conocimiento, capacidad predictiva y baja resistencia de estas aleaciones a este tipo de ataque. Son la comprensión, predicción y el control, los retos claves para el diseño de facilidades, operación y consecuente garantía de la integridad (Kermani \& Morshed, 2003).

En los pozos productores del Campo A, las concentraciones de Sulfuro de Hidrógeno - $\mathrm{H}_{2} \mathrm{~S}$ son bajas, el fluido producido contiene un alto porcentaje de agua, 2 millones 200 barriles de fluido por día y 65 mil barriles de aceite BOPD. Los fluidos producidos presenta gran cantidad de arena, que ocasiona problemas de abrasión (erosión) en las tuberías de producción, y por lo tanto, deja vulnerable la tubería ayudando a que la corrosión sea más rápida al producirse a altas velocidades.
Los problemas de corrosión que se presentan en los pozos productores del Campo A, son ocasionados principalmente por la presencia de altas concentraciones Dióxido de Carbono - $\mathrm{CO}_{2}$ y la producción de arena.

Número de Intervenciones que se realizan a los pozos afectados en el Campo A: Cuando se presume que algún pozo pueda tener corrosión, es decir para las zonas donde se produce gas, las intervenciones se realizan una vez por año, encontrándose tuberías corroídas en este tiempo.

En promedio, los tiempos de vida de los equipos de fondo están alrededor de los 700 días, tiempo en que normalmente se realizarían las intervenciones si no se presentara corrosión. El costo asociado a una intervención para un pozo con completamiento con empaque puede estar alrededor de los 300 mil dólares.

Y si es un completamiento sin empaque, la intervención puede estar alrededor de los 200 mil dólares. El empaque se utiliza para los pozos que tiene alta producción de gas y los que están separados en los límites geográficos del Campo, para prevenir fugas en los ataques vandálicos.

Vida Útil de la Tubería de Producción de Fondo Tradicional que se está utilizando actualmente: Las Tuberías de Producción Tradicionales en el Campo A tienen un tiempo de vida útil entre 700 y 1.500 días, cuando las condiciones del yacimiento presentan ambientes corrosivos. Si la corrosión es muy crítica dichas tuberías pueden tener una vida útil promedio de un año.

En pozos sin problemas de corrosión la Tubería de Producción Tradicional presenta una vida útil de 2.000 días. En el Campo A se han tenido casos de pozos excepcionales con tuberías muy longevas pero están ubicados en zonas donde no se produce gas y el yacimiento no tiene condiciones corrosivas.

Cantidad de Barriles Diarios que se dejan de producir debido a la actividad de corrección y mitigación de los daños causados por la Corrosión: En promedio se producen en el Campo A por pozo alrededor de 400 barriles por día (es decir, se tienen pozos muy buenos de hasta 1.000 barriles por día, pero también existen otros pozos de muy baja producción hasta 100 barriles por día).

Una intervención desde que el pozo falla hasta que lo ponen en línea, tarda alrededor de once (11) días. Cuando el pozo es muy bueno hacen lo posible para que se pueda intervenir en un tiempo menor. 
Dado lo anterior la cantidad de barriles que se dejan de producir en una intervención es alrededor de 4.400 barriles.

\section{MITIGACIÓN DE LA CORROSIÓN EN LA INDUSTRIA DEL PETRÓLEO Y GAS}

Los desafíos de la corrosión en un campo petrolero no son fenómenos estáticos, debido a que las características de fluidos cambian con el tiempo. Lo anterior, da lugar a sistemas cada vez menos sensibles a los programas de mitigación de la corrosión establecidos (Champion Technologies, 2012). En el ámbito de control de la corrosión y la prevención en la industria del petróleo y el gas, se han implementado técnicas tales como la protección catódica y anódica, la correcta selección de materiales, la dosificación química, y la aplicación de revestimientos internos y externos (Nalli, 2010).

Capas Protectoras de Revestimiento Interno: Es una capa de protección o barrera en el material para evitar el contacto directo con el fluido del proceso mejorando la vida del material y del equipo. La capa de barrera puede ser la pintura, un revestimiento o forro metálicos. Actualmente también existen revestimientos no metálico como la fibra de vidrio, escamas de vidrio, epoxi y el caucho, los cuales son utilizados normalmente en equipos como separadores, tanques knock-out y tanques de almacenamiento. Los revestimientos de níquel, zinc y cadmio, son preferidos en determinados componentes como bridas y pernos (Nalli, 2010).

\section{VENTAJAS TÉCNICAS DE UTILIZAR LAS NUEVAS TECNOLOGÍAS EN CONTRASTE CON AQUELLAS ACTUALMENTE UTILIZADAS EN EL CAMPO}

Dadas las nuevas tecnologías presentes en el mercado para tubería revestida internamente, las ventajas técnicas que se tendrían serían:

- Fácil Movilidad de los Fluidos: La capacidad de las tuberías revestidas internamente se aumenta entre un $14 \%$ y $21 \%$ en comparación con una tubería sin revestimiento, lo anterior se debe a que al tener la tubería un recubrimiento interior, los fluidos producidos tendrán un medio que ayuda a la movilidad de los mismos (Documento Técnico de Pipeline International, 2010).
Mediante el uso de tubería revestida, se mejora la eficiencia del flujo incrementando el volumen del flujo producido y reduciendo el consumo de energía. La fácil movilidad de los fluidos representa un ahorro directo en el número de caballos de fuerza y la energía necesaria para impulsar el fluido producido (Huggins, 2010).

- Protección a la Corrosión: Un informe completado en 2006 por John Grover of BJ Process \& Pipeline Services señaló que un estimado de $157.000 \mathrm{~kg}$ de residuos de corrosión se eliminan de una sección de 161 kilómetros de diámetro de la tubería de 36 pulgadas con un espesor de pared de $14,3 \mathrm{~mm}$, sólo tres meses después de la inmersión total en agua de mar tropical (Documento Técnico de Pipeline International, 2010).

Mediante el uso de tuberías de producción revestida, se retarda la aparición de corrosión en el corto tiempo, eliminado la necesidad de realizar trabajos de pre-comisionamiento y workovers innecesarios.

El revestimiento interno reduce la superficie sometida a la corrosión por picadura sólo a aquellos lugares donde el recubrimiento se daña y no es reparado. Ahora es técnicamente posible instalar un revestimiento interno con cero defectos (Huggins, 2010).

- Protección a la Abrasión: Cuando se tiene un yacimiento donde los fluidos producidos contienen gran cantidad de arena, dicha arena produce abrasión en las tuberías de producción, dejándolas expuestas. La tubería de producción revestida es resistente a la abrasión, adicionalmente sirve como un aislante para mitigar el desgaste de tubos de la bomba y PCP de los pozos (varilla y/o guías de la varilla).

- Puesta en marcha e Inspección Simplificada: Después de haber realizado una prueba hidrostática, una tubería revestida internamente se secará más rápido que una tubería no recubierta, lo que significa que se podrá poner en marcha más rápidamente y de una manera más fácil (Documento Técnico de Pipeline International, 2010).

El revestimiento interno retrasará la primera fuga y en última instancia, reduce el número total de las reparaciones realizadas en la tubería durante su vida útil. 
- Reducción de los Costos de la Energía en el Bombeo: Al tener la tubería un recubrimiento interior, los fluidos producidos tendrán un medio que opone menos resistencia al movimiento y por tanto ayuda a la movilidad de los fluidos en la misma. Lo anterior, acarrea que la bomba requiera menos energía para impulsar los fluidos hasta superficie, lo cual reduce el consumo de energía con respecto a producir los fluidos con completamientos de tuberías sin revestir. Estos costos energéticos reducidos pueden proporcionar un retorno de la inversión financiera dentro de tres a cinco años de servicio.

- Reducción de la Tasa de Falla: Reducir sustancialmente la tasa de incidencia y por lo tanto el costo de la falla catastrófica que resulta en lesiones personales, daños al medio ambiente y la limpieza, la pérdida de ingresos de producción y el de relaciones públicas / daños imagen corporativa negativa (Huggins, 2010).

Adicional a las características arriba expuestas, las tuberías de producción revestidas presentan ventajas puntuales tales como que, son químicamente inertes ante los fluidos de estimulación, además de flexibles, ligeras, reciclable, permiten el almacenamiento in situ (por ejemplo en zonas costeras) con un mínimo de riesgo de corrosión, y no añade más impurezas al fluido producido.

\section{PROPUESTA TÉCNICA PARA LA MEJORA}

La propuesta técnica para la mejora de las facilidades de producción del Campo A es cambiar la tubería de producción tradicional por la tubería de producción revestida, a fin de reducir costos operacionales y mitigar riesgos ambientales y de seguridad industrial.

De las diferentes posibilidades de tuberías de producción revestida (presentes actualmente en el mercado y probadas en casos exitosos y campos similares al Campo A), después de realizar el análisis de las características de cada una de ellas y teniendo en cuenta la amplia experiencia de la compañía DUOLINE en la fabricación, aplicación y mantenimiento de tuberías revestidas, adicionalmente dadas las pruebas realizadas por dicha compañía en campos similares al Campo A, la tecnología ofrecida (con mayores beneficios técnicos que la competencia), su amplia gama de aplicación y que dicha compañía tiene presencia en Colombia, se recomienda seleccionar como la mejor opción a ser implementada en los próximos años en el Campo A, las tuberías revestidas cotizadas por dicha compañía como se muestra a continuación:

- Coating Tubing: Size 3 1/2", Weigth: 9,3 LB/FT, Specification: API 5CT, Grade J55, End Type: EUE 8RD, DDP Campo.

- Coating Tubing: Size 2 7/8", Weigth: 6,5 LB/FT, Specification: API 5CT, Grade N80, End Type: EUE 8RD. DDP Campo.

\section{INGENIERÍA DE VALOR}

Las variables que se tuvieron en cuenta para el cálculo económico - financiero de la propuesta se muestran en la Tabla 4.

Tabla 4. Variables para el cálculo económico

\begin{tabular}{|c|c|}
\hline Variable & Valores \\
\hline $\begin{array}{l}\text { Profundidad promedio } \\
\text { pozos }\end{array}$ & $7.600 \mathrm{ft}$ \\
\hline $\begin{array}{l}\text { Costo de una } \\
\text { Intervención }\end{array}$ & $\begin{array}{c}\text { Con empaque: USD } \$ 300.000 \text { y sin } \\
\text { empaque: USD } \$ 200.000\end{array}$ \\
\hline $\begin{array}{l}\text { Duración de una } \\
\text { Intervención }\end{array}$ & Entre 1 y 2 semanas, promedio: 11 días \\
\hline $\begin{array}{l}\text { Producción Diaria } \\
\text { promedio }\end{array}$ & 400 barriles \\
\hline $\begin{array}{l}\text { Precio del Barril de } \\
\text { Petróleo }\end{array}$ & USD $\$ 42$ \\
\hline $\begin{array}{l}\text { Cantidad de } \\
\text { intervenciones }\end{array}$ & $\begin{array}{c}\text { Para la Tubería de Producción } \\
\text { Tradicional se deben hacer } \\
\text { Intervenciones cada dos años (cada } 730 \\
\text { días) mientras que para la Tubería de } \\
\text { Producción Revestida se hacen cada } \\
\text { seis años (2.190 días). }\end{array}$ \\
\hline $\begin{array}{c}\text { Vida útil de la Tubería } \\
\text { de Producción } \\
\text { Tradicional }\end{array}$ & $\begin{array}{c}\text { De } 700 \text { a } 1.500 \text { días. Si la corrosión es } \\
\text { crítica } 1 \text { año }\end{array}$ \\
\hline $\begin{array}{c}\text { Vida útil de la Tubería } \\
\text { de Producción } \\
\text { Revestida }\end{array}$ & $\begin{array}{c}\text { De } 1.700 \text { a } 4.000 \text { días, sin utilizar } \\
\text { ningún otro medio de prevención de la } \\
\text { corrosión }\end{array}$ \\
\hline
\end{tabular}

Los costos de las Tubería de Producción Tradicional y la Tubería de Producción Revestida seleccionadas según las características técnicas se muestran en la Tabla 5 y la Tabla 6. 
Tabla 5. Costo de la Tubería de Producción Tradicional

\begin{tabular}{|c|c|c|}
\hline Ítem & Descripción del Objeto & $\begin{array}{l}\text { Valor Unitario USD } \\
\qquad(\mathrm{Ft})\end{array}$ \\
\hline 1 & $\begin{array}{l}\text { Tubing: Size } 3 \text { 1/2", Weigth: 9,2 LB/FT, Specification: API 5CT, Grade J55, Process: Seamless, End } \\
\text { Type: EUE 8RD, Range: R2 con Cuellos Instalados, DDP Campo }\end{array}$ & $\$ 6,49$ \\
\hline 2 & $\begin{array}{l}\text { Tubing: Size } 27 / 8 \text { ", Weigth: } 6,5 \text { LB/FT, Specification: API 5CT, Grade N80, Process: Seamless, End } \\
\text { Type: EUE 8RD, Range: R2 con Cuellos Instalados, DDP Campo }\end{array}$ & $\$ 4,88$ \\
\hline
\end{tabular}

Tabla 6. Costo de la Tubería de Producción Revestida

\begin{tabular}{|c|c|c|}
\hline Ítem & Descripción del Objeto & $\begin{array}{l}\text { Valor Unitario USD } \\
\text { (Ft) }\end{array}$ \\
\hline 1 & $\begin{array}{l}\text { Coating Tubing: Size } 3 \text { 1/2", Weigth: 9,3 LB/FT, Specification: API 5CT, Grade J55, End Type: EUE } \\
\text { 8RD, DDP Campo }\end{array}$ & $\$ 20,77$ \\
\hline 2 & $\begin{array}{l}\text { Coating Tubing: Size } 2 \text { 7/8", Weigth: 6,5 LB/FT, Specification: API 5CT, Grade N80, End Type: EUE } \\
\text { 8RD. DDP Campo }\end{array}$ & $\$ 17,28$ \\
\hline
\end{tabular}

Tomado como base la Tubería de Producción Tradicional de la Empresa Technical Petroleum Services S.A. "TUBING: SIZE 3 1/2", WEIGTH: 9,2 LB/FT, SPECIFICATION: API 5CT, GRADE J55, PROCESS: SEAMLESS, END TYPE: EUE 8RD, RANGE: R2 CON CUELLOS INSTALADOS, DDP CAMPO”, el costo de la Tubería de Producción Tradicional es de: USD $\$ 50.000$.

Tomado como base la Tubería de Producción Revestida de la Empresa DUOLINE TECHNOLOGIES "COATING TUBING: SIZE 3 1/2”, WEIGTH: 9,3 LB/FT, SPECIFICATION: API 5CT, GRADE J55, END TYPE: EUE 8RD, DDP CAMPO”, el costo de la Tubería de Producción Revestida es: USD \$160.000.
La Inversión Inicial es: USD \$110.000.

Las pérdidas de producción durante una Intervención son: USD \$185.000.

El costo de una Intervención con empaque es: USD $\$ 300.000$.

La Tasa de descuento se tomó del 12\% y el número de periodos de 6 años.

En la Tabla 7 se muestra el cálculo del Valor Presente Neto del proyecto de cambiar la tubería tradicional por tubería revestida.

Tabla 7. Cálculo del Valor Presente Neto (VPN) del Proyecto

\begin{tabular}{|c|c|c|c|}
\hline \multicolumn{4}{|c|}{ Valor Presente Neto (VPN) } \\
\hline No. & FNE & $(1+i)^{n}$ & FNE $/(1+i)^{n}$ \\
\hline 0 & $(110.000,00)$ & & $(110.000,00)$ \\
\hline 1 & 0 & 1,12 & 0 \\
\hline 2 & $485.000,00$ & 1,25 & $386.639,03$ \\
\hline 3 & 0 & 1,40 & 0 \\
\hline 4 & $485.000,00$ & 1,57 & $308.226,27$ \\
\hline 5 & 0 & 1,76 & 0 \\
\hline 6 & 0 & 1,97 & 0 \\
\hline \multicolumn{3}{|c|}{ TOTAL } & $584.865,30$ \\
\hline \multicolumn{3}{|c|}{$\mathbf{V P N}=$} & $\$ 584.865,30$ \\
\hline
\end{tabular}


En la Tabla 8 se muestra el cálculo de la Tasa Interna de Retorno del Proyecto de cambiar la tubería tradicional por tubería revestida.

Tabla 8. Cálculo de la Tasa Interna de Retorno (TIR) del Proyecto

\begin{tabular}{|cc|}
\hline \multicolumn{2}{|c|}{ Tasa Interna de Retorno $(\mathrm{TIR})$} \\
\hline Tasa de Descuento & VPN \\
\hline $0 \%$ & $\$ 860.000,00$ \\
\hline $12 \%$ & $\$ 584.865,30$ \\
\hline $24 \%$ & $\$ 410.568,83$ \\
\hline $36 \%$ & $\$ 293.989,43$ \\
\hline $48 \%$ & $\$ 212.507,64$ \\
\hline $60 \%$ & $\$ 153.458,25$ \\
\hline $72 \%$ & $\$ 109.355,05$ \\
\hline $84 \%$ & $\$ 75.566,45$ \\
\hline $96 \%$ & $\$ 49.113,25$ \\
\hline $108 \%$ & $\$ 28.013,69$ \\
\hline $120 \%$ & $\$ 10.910,46$ \\
\hline $132 \%$ & $\mathbf{( \$ 3 . 1 5 0 , 1 7 )}$ \\
\hline $144 \%$ & $\mathbf{( \$ 1 4 . 8 5 3 , 6 5 )}$ \\
\hline TIR $=$ & $\mathbf{1 2 9 , 1 0 8 4 \%}$ \\
\hline
\end{tabular}

\section{MITIGACIÓN DE RIESGOS AMBIENTALES Y DE SEGURIDAD INDUSTRIAL}

Mediante la implementación del proyecto se incrementará la seguridad industrial al personal de campo y se reducirán la tasa de accidentes que ocasionen daños ambientales graves. Adicionalmente se reducirá la tasa de incidencias y fallas inesperadas de la tubería que pudieran ocasionar:

- Fugas de crudo y gas: tales como derrames de petróleo, contaminación del aire, suelo, subsuelo y el agua superficial y subterránea (impotable).

- Lesiones personales: ocasionadas por reventones catastróficos al no soportar la presión (dada la corrosión).

- Problemas respiratorios en los empleados por inhalación de gases que se escapen sin conocimiento alguno, dada la corrosión en las tuberías.

Para la compañía en caso de desastre se pudiera traducir en pérdidas en ingresos de producción, daños en las relaciones públicas y la repercusión negativa en la imagen y acciones.

\section{CONCLUSIONES}

Tomando como base las investigaciones y proyectos realizados en el Campo A, se realizó la evaluación actual de las facilidades de producción del Campo A, entre las cuales se demostró que actualmente en el Campo el $20 \%$ de los pozos productores ( 70 pozos) presentan problemas de corrosión principalmente debido a la alta producción de arena que genera abrasión y a la presencia de Dióxido de Carbono $\mathrm{CO}_{2}$. Adicionalmente, se obtuvo información necesaria para realizar la evaluación técnica y económica del proyecto, tales como los tiempos de vida útil y costos de la tubería tradicional y revestida, tiempos y costos de una intervención, la producción actual del campo en los pozos vecinos donde se desarrolló el proyecto, el plan de desarrollo del campo y las características geográficas y técnicas del sitio donde se desarrolló el proyecto, entre otros.

Teniendo en cuenta el análisis técnico realizado, se observó que al utilizar tubería de producción revestida se podrán obtener las siguientes ventajas técnicas, en comparación con la tubería tradicional:

a. Se retarda la aparición de corrosión en el corto tiempo, eliminando la necesidad de realizar trabajos de pre-comisionamiento y workovers innecesarios, lo que acarrea que la producción sea mayor a la esperada con un completamiento de tubería tradicional, debido a la continua e ininterrumpida producción de crudo en un periodo no menor a seis años.

b. Se reducen los problemas de abrasión de la tubería, dado que la tubería de producción revestida es resistente a la abrasión.

c. Sirve como un aislante para mitigar el desgaste de tubos de la bomba ESP y PCP de los pozos (varilla y/o guías de la varilla), dado que no hace que las facilidades se esfuercen más de lo necesario y por tanto aumentan la vida útil de dichas facilidades.

d. Ayuda a la fácil movilidad de los fluidos, dado que al tener la tubería un recubrimiento interior, los fluidos producidos tendrán un medio que ayuda a la movilidad de los mismos, aumentando entre un $14 \%$ y $21 \%$ en comparación con una tubería sin revestimiento.

e. Se mejora la eficiencia del flujo, incrementando el volumen del fluido producido y reduciendo el consumo de energía. La fácil movilidad de los fluidos representa un ahorro directo en el número de caballos de fuerza y la energía necesaria para impulsar el fluido producido. 
f. En combinación con otros métodos como los inhibidores químicos, aumenta considerablemente la esperanza de vida de la tubería.

g. Adicional a las características arriba expuestas, las tuberías de producción revestidas presentan ventajas puntuales tales como que, son químicamente inertes ante los fluidos de estimulación, además de flexibles, ligeras, reciclable, permiten el almacenamiento in situ (por ejemplo en zonas costeras) con un mínimo de riesgo de corrosión, y no añade más impurezas al fluido producido.

h. El espectro de uso de las tuberías de producción se amplía, dado que resisten más condiciones adversas (yacimientos con alto porcentaje de $\mathrm{CO}_{2}, \mathrm{H}_{2} \mathrm{~S}$ y producción de agua y arena).

i. Reduce sustancialmente la tasa de incidencia y por lo tanto el costo de la falla catastrófica que resulta en lesiones personales, daños al medio ambiente, la pérdida de ingresos de producción, daños en las relaciones públicas y por consiguiente repercusión negativa de la imagen corporativa.

Teniendo en cuenta el análisis económico realizado, se observa que al utilizar tubería de producción revestida se podrán obtener los siguientes ahorros, en comparación con la tubería tradicional:

a. Debido a la reducción en el número de intervenciones a los pozos, se ahorran los costos de dos intervenciones en los seis años de la vida útil de la Tubería Revestida, (alrededor de USD \$300.000 por cada intervención), lo anterior, dado que para la Tubería de Producción Tradicional se deben hacer Intervenciones cada dos años (cada 730 días) mientras que para la Tubería de Producción Revestida se hacen cada seis años (2.190 días).

b. Las diferidas de producción debidas a la intervención de un pozo que tenga una duración de 11 días costarían a la compañía un total de USD \$185.000 por cada intervención. Debido a que se reduce el número de intervenciones, dicha producción diferida ya no se perdería.

c. Al realizar el cálculo del VPN en el cual se compara utilizar la Tubería de Producción Tradicional versus la Tubería de Producción Revestida en el completamiento de un pozo nuevo y proyectarlo a un periodo de seis años, se obtiene que el valor presente del flujo de caja futuro es de USD $\$ 584.865,30$ por pozo nuevo, lo es un indicativo de que nuestra propuesta, desde el punto de vista financiera será rentable. Lo anterior, debido a que mediante la aplicación de la propuesta, se generará suficiente dinero para que la inversión sea recuperada y se genere una ganancia.

d. Al realizar el cálculo de la TIR en el cual se compara utilizar la Tubería de Producción Tradicional versus la Tubería de Producción Revestida en el completamiento de un pozo nuevo y proyectarlo a un periodo de seis años, se obtiene que la rentabilidad promedio de la inversión por pozo es de $129,1084 \%$, lo cual hace que el proyecto sea muy atractivo para el Operador del Campo A.

\section{AGRADECIMIENTOS}

Un agradecimiento especial al equipo de tutores y coordinadores de la Universidad de Viña del Mar, especialmente a nuestros Tutores Marco Antonio Calle Martínez y Paulina Monares Ramírez y al Profesor Informante Mauricio Javier Rodríguez Sepúlveda por guiar, respaldar, apoyar y contribuir en nuestras ideas para la realización del Trabajo de Grado de la Maestría en Gestión en la Industria de los Hidrocarburos.

A la Universidad de Viña del Mar, por brindarnos un espacio propicio para el aprendizaje y desarrollo de éste trabajo y así contribuir con nuestro proceso de formación profesional a través de la educación virtual.

A la Universidad Surcolombiana, especialmente a la profesora Isabel Cristina Gutiérrez de Dussán, Vicerrectora Académica, por el aval dado para desarrollar nuestro trabajo de grado y brindarnos todo su respaldo.

A la empresa Occidental de Colombia, LLC - OXY por suministrarnos la información necesaria para el desarrollo del proyecto, principalmente a Luis Carlos Villamizar Delgado, Ingeniero de Operaciones de la empresa.

\section{REFERENCIAS}

1. Amorocho, L., D., M. (2006). Análisis técnico - económico del retiro de válvulas de seguridad de fondo y panel OTIS en pozos del Campo Caño Limón (tesis de pregrado). Universidad Industrial de Santander, Bucaramanga, Colombia. Recuperado de http://repositorio.uis.edu.co/jspui/ bitstream/123456789/1049/2/119508.pdf.

2. Castillo, V., E., (2005), Evaluación de las zonas productoras abandonadas de pozos de los campos Caño Limón y Caño Verde que presentan cortes actualesdeaguasuperioresal97\%(tesisdepregrado). Universidad Industrial de Santander, Bucaramanga, Colombia. Recuperado de http://repositorio.uis.edu. 
co/jspui/bitstream/123456789/1010/2/116220.pdf.

3. Champion Technologies. (2012). Corrosion mitigation for complex environments. Champion Technologies, Houston.

4. Colque, A., G., (2012), Diseño de tuberías de producción, Universidad de Aquino de Bolivia. Recuperado de: http://es.scribd.com/ doc/90358531/DISENO-DE-TUBERIAS-DEPRODUCCION\#download.

5. Cuadrado, N., F., G. \& Prada, T., J., W. (2005). Evaluación de la corrosión - desgaste entre los acoples de las varillas de pozo y la tubería de producción en sistemas de levantamiento artificial de petróleo (tesis de pregrado). Universidad Industrial de Santander, Bucaramanga, Colombia. Recuperado de http://repositorio.uis.edu.co/jspui/ bitstream/123456789/1418/2/116926.pdf

6. Crolet, J. L. \& Bonis, M. (1983). pH Measurements in Aqueous $\mathrm{CO}_{2}$ Solutions under High Pressure and Temperature. Corrosion, 39(2). p. 39-46.

7. Documento Técnico de PDVSA. (2008). Mecanismos de corrosión en sistemas de producción del campo Dación.

8. Documento Técnico de Pipeline International. (2010). Inside and out: benefits of pipeline coating.

9. Guevara, M., M., F. (2010). Estimación del potencial incrustante y corrosivo en pozos productores y líneas de transferencia del Campo Dación, San Tomé (tesis de pregrado). Universidad de Oriente, Puerto la Cruz, Venezuela. Recuperado de http://ri.biblioteca. udo.edu.ve/bitstream/123456789/2802/1/076TESIS.IQ.pdf

10. Harrop, D., Martin, J. W., \& White, C. W. (1988).
The Use of Synthetic Environments for Corrosion Testing. ASTM International. ASTM STP970-88.

11. Huggins J.A. (2010). What is important about internal field joint coating, re-impreso por www. worldpipelines.com.

12. Jaspe, R., U., R. (2006). Estudio de la resistencia a la corrosión por $\mathrm{CO}_{2}$ de aceros de tuberías 3\% Cr para pozos petroleros (tesis de pregrado). Universidad Simón Bolívar, Sartenejas, Venezuela. Recuperado de http://159.90.80.55/tesis/000142075.pdf.

13. Kermani, M. P., \& Morshed, A. (2003). Carbon Dioxide Corrosion in Oil and Gas. Corrosión, 59(8).

14. Kermani, M. B., \& Smith, L. M. (1997). CO Corrosion Control in Oil and Gas ProductionDesign Considerations. European Federation of Corrosión Publication, (23).

15. Lusk, D., Gupta, M., Boinapally, K., \& Cao, Y. (2008). Armoured against corrosion. Hydrocarb Eng 13:115-118.

16. Nalli, K. (2010). Corrosion and its mitigation in the oil and gas industry. An overview. PM-Pipeliner Report.

17. Papavinasam, S., Doiron, A., Panneerselvam, T., \& Revie, R. W. (2007). Effect of Hydrocarbons on the internal corrosion of oil and gas pipelines. Corrosion, 63(7), 704.

18. Popoola, L. T., Grema, A. S., \& Latinwo, G. K., (2013). Corrosion problems during oil and gas production and its mitigation. International Journal of Industrial Chemistry 4:35.

19. Roberge, P. R. (2000). Handbook of corrosion engineering. McGraw-Hill, New York.

Recepción: 26 de febrero de 2016

Aceptación: 16 de abril de 2016 